\title{
INTERVENÇÃO NA DEPRESSÃO GERIÁTRICA ATRAVÉS DA REMINISCÊNCIA
}

\author{
Intervention in Geriatric Depression Through Reminiscence
}

\author{
Daniela C. Gonçalves ${ }^{1}$ \\ Ignácio Martín ${ }^{2}$
}

\section{Resumo}

Actualmente, a depressão é a problemática em termos de saúde mental com maior prevalência entre as pessoas idosas. Considerando a evolução verificada nas últimas décadas, as intervenções farmacológicas e psicoterapêuticas, como a cognitivo-comportamental, mostraram ser instrumentos eficazes de tratamento. Não obstante, outras estratégias, consideradas como não-tradicionais, devem também ser consideradas como estratégias válidas de intervenção na depressão das pessoas idosas. Assim, têm despertado um assinalado interesse, em parte por não terem as limitações das intervenções farmacológicas, como os efeitos secundários, e cognitivo-comportamentais, como o elevado grau de exigência cognitiva. A mais conhecida deste conjunto de estratégias é a terapia da reminiscência. O propósito deste artigo é analisar a reminiscência enquanto estratégia de intervenção junto de idosos com sintomatologia depressiva por meio duma revisão da literatura acerca deste tópico tanto em termos de estudos de sínteses, nomeadamente a meta-análise, e estudos empíricos desenvolvidos nos últimos dez anos. A terapia da reminiscência é apresentada enquanto instrumento terapêutico e avaliada a sua eficácia enquanto estratégia de intervenção precoce na sintomatologia depressiva de idosos, assim como a sua função na capacidade que tem de atribuição de significado de vida e preparação para a morte. Como conclusão, são destacadas algumas linhas de investigação a realizar neste âmbito.

Palavras-chave: Depressão; Pessoas idosas; Terapia da reminiscência.

\footnotetext{
Docente universitária da Universidade Católica Portuguesa (Porto). Mestre em Psicologia Clínica e Membro da Unidade de Investigação e Formação sobre Adultos e Idosos (UnIFAi).e-mail: daniela@unifai.net

2 Docente universitário da Universidade de Aveiro na Secção Autónoma de Saúde. Doutor em Ciências Biomédicas e Membro da Unidade de Investigação e Formação sobre Adultos e Idosos (UnIFAi) - Secção Autónoma de Ciências da Saúde. Universidade de Aveiro - Campo Universitário de Santiago. e-mail: jmartin@cs.ua.pt
} 


\section{Abstract}

Depression is the most prevalent mental illness amongst the elderly. Considering the amount of work produced on the last decades, the pharmacological interventions and cognitive-behavioural psychotherapies have shown to be effective treatments. Nevertheless, other strategies considered non-traditional should also be measured as valid strategies of intervention for depressive older people because of the fact of these therapies not having the limitations of the pharmacological and cognitivebehavioural interventions, such as side effects and cognitive demands. From this group of strategies the reminiscence therapy is the most used. The purpose of this article is to analyse the reminiscence therapy as a strategy of intervention amongst elders that have symptoms of depression, by using a literature review about this topic, namely meta-analysis and empirical studies developed over the past ten years. The reminiscence therapy is a therapeutic instrument and as such its effectiveness is evaluated in different ways. It is evaluated as an early intervention strategy in the elderly that suffer from depressive symptoms, as well as its capacity to bring meaning to life and preparation for death. In conclusion, we propose some additional research variables in this domain.

Keywords: Depression; Elderly; Reminiscence therapy.

A depressão é o problema saúde mental mais comum entre as pessoas idosas, sendo as suas consequências mais frequentes localizadas na esfera funcional, cognitiva e emocional, fomentando o risco de quedas, má nutrição e maior probabilidade de infecções (Blazer, 2002a; 2003; Bowling \& Farquhar, 1996; Baldwin \& Wild, 2004).

Apesar das limitações existentes quanto à prevalência das doenças mentais em Portugal, as estimativas indicam que cerca de metade das pessoas idosas institucionalizados manifesta algum tipo de sintomatologia depressiva (Costa, 2005). Considerando o aumento progressivo do número de idosos, este é indubitavelmente um problema de cariz sanitário, social e económico bastante grave (INE, 2002; Katona \& Shankar, 1999, 2004).

Conquanto estes números sejam já preponderantes, os estudos apontam para a existência de muitos casos de depressão geriátrica não identificados pelos profissionais de saúde, não sendo diagnosticados ou sendo diagnosticados como outra patologia (Jones, 2003; Lee, Volans \& Gregory, 2003; Voyer \& Martin, 2003; Bergdahl et al., 2005). O subdiagnóstico no âmbito da saúde mental em idosos é simultaneamente explicado pelas características associadas às pessoas idosas e aos prestadores de cuidados de saúde. Verifica-se por parte das pessoas idosas relutância em pedir ajuda, ausência de identificação dos seus sinais e dificuldade em aceder a cuidados de saúde (Jones \& Beck-Little, 2002). Por parte dos profissionais de saúde, existem muitas vezes lacunas na sua formação específica, o que dificulta o processo de identificação de sintomas e diagnóstico
(APA, 2004; \& Murphy, 2000). Ambas as situações conduzem a um quadro de subdiagnóstico e consequente ausência de intervenção na sintomatologia depressiva.

O desenvolvimento de técnicas e estratégias de detecção e avaliação de sintomas, bem como de intervenção específicas para idosos, é também condicionado pela inexistência de um corpo teórico integrado e significativo sobre o processo de envelhecimento. Esta lacuna é constatada através da ausência de referência a este período da vida numa parte substancial das teorias do desenvolvimento (Hepple, 2004). Poucos estudos incidiram sobre as potencialidades do desenvolvimento humano após a adolescência, negligenciando as transições que acontecem e as potencialidades da intervenção psicoterapêutica nesta fase de vida, na promoção da adaptação a essas mesmas transições.

As consequências do subdiagnóstico da depressão nas pessoas idosas são variadas, com ramificações económicas, por aumento da dependência e das despesas associadas; sociais, em função das necessidades de prestação de cuidados, formais e informais; sanitárias, devido à demanda de tratamentos; e éticas, dadas as repercussões no bem-estar do idoso (APA, 2004; \& Costa, 2005). Deste modo, concluímos que a depressão no idoso é um problema grave, com impacto negativo na qualidade de vida e agravamento de doenças (Baldwin \& Wild, 2004), que conduz a uma sobreutilização dos serviços e morte precoce (Frazer, Christensen \& Griffiths 2005). 
Os dados relativos ao prognóstico da intervenção com idosos variam consoante o contexto em que são recolhidos, nomeadamente entre amostras rurais e urbanas ou idosos a viver em comunidade ou institucionalizados (Baldwin, \& Wild, 2004; Form, 2000). Os factores de risco associados às pessoas idosas, entre os quais a presença de comorbilidade fisiológica, desempenham um papel preponderante na eventual recuperação e condicionam os dados encontrados (Frazer et al., 2005; Shah \& Oxey, 2001; Woods, 1999).

Os tratamentos medicamentosos, através dos antidepressivos, possuem já uma eficácia comprovada na intervenção na depressão geriátrica (Mulsant et al., 2006; Taylor \& Doraiswamy, 2004). A psicoterapia, nomeadamente a terapia cognitivocomportamental, apresenta também taxas de sucesso consideráveis para intervir junto de pessoas idosas com quadros depressivos (Scogin, Welsh, Hanson, Stump \& Coates 2005).

Contudo, apesar da sua eficácia, ambas as estratégias apresentam inconvenientes. A medicação antidepressiva pressupõe o surgimento de efeitos secundários, como maior probabilidade de quedas, probabilidade elevada de recaída, após retirada da medicação e necessidade de adaptação da dose ao idoso, correndo o risco de doses inadequadas ou de interacção com outras patologias existentes (Belsky, 1999; Jones \& Beck-Little, 2002; Frazer et al., 2005; Baldwin \& Wild, 2004). A psicoterapia, enquanto metodologia estruturada de intervenção, acarreta gastos consideráveis, mas sobretudo exige determinadas competências cognitivas por parte do idoso (Douglas, James \& Ballard, 2004; Laidlaw, 2001). Adicionalmente, sendo que é uma estratégia generalizada, pode não contemplar as necessidades do idoso, isto é, os seus objectivos podem não fazer sentido no momento específico de vida em que o idoso se encontra (Blazer, 2002b; Hepple, 2004).

Com o intuito de ultrapassar as dificuldades inerentes à utilização de estratégias generalizadas com idosos e de evitar os efeitos secundários associados à medicação, foi desenvolvido um conjunto de estratégias de intervenção com idosos, passíveis de utilização em diversos quadros de funcionamento cognitivo e emocional. Considerando que as estratégias podem ser utilizadas em diferentes contextos, mesmo na ausência de patologias psicológicas, são habitualmente denominadas de intervenções precoces para a promoção do bem-estar (Scott \&
Clare, 2003). Entre esta tipologia de intervenções, destacam-se a Orientação à Realidade, a Terapia de Validação e sobretudo Terapia da Reminiscência (TR).

A Orientação à Realidade e a Terapia da Validação são estratégias utilizadas com idosos com défices cognitivos, habitualmente do foro demencial, com o intuito de promover o funcionamento diário (Beaver \& Miller, 1998; Grasel, Wiltfang \& Kornhuber, 2003). Conquanto as premissas destas estratégias sejam opostas (a orientação à realidade postula que o idoso seja continuamente orientado, no tempo, lugar e pessoa; a terapia da validação perspectiva a confusão como um mecanismo de coping que não deve ser confrontado), ambas pretendem diminuir as consequências dos sintomas demenciais, promovendo a adaptação do idoso ao novo modo de funcionamento (Douglas et al., 2004).

Considerando que o objectivo deste artigo é a análise e compreensão da eficácia da reminiscência como estratégia de intervenção na depressão geriátrica, vamos em seguida realizar uma exploração mais aprofundada do conceito de reminiscência. Num primeiro momento, conceptualizamos a reminiscência enquanto processo inerente ao desenvolvimento humano, utilizada de modo tácito para resolução de problemas e integração de experiências. Em segundo lugar, examinamos os fundamentos teóricos da reminiscência como estratégia de intervenção, comentando os princípios que antecederam o seu surgimento, as definições existentes e os objectivos habitualmente pretendidos com a sua utilização. Por fim, analisamos alguns dos estudos realizados no âmbito da intervenção na depressão geriátrica, diferenciando os estudos no âmbito da revisão de literatura e das meta-análises e os estudos empíricos.

\section{A Reminiscência}

A reminiscência é um processo natural e espontâneo, inerente ao ser humano, de evocação das experiências significativas ocorridas no passado (Jonsdottir, Steingrimsdottir \& Tryggvadottir, 2001). Evocamos e contamos as histórias da nossa vida, a nós próprios e aos outros, com o intuito de recordarmos experiências e acontecimentos passados, habitualmente com conotação positiva ou negativa (Aberg, Sidenvall, Hepworth, O’Rilley \& Lithell, 2005). Recordar situações que vivemos, de 
modo individual ou interaccional, permite a análise do passado, a compreensão de mudanças, a adaptação a transições, a aquisição de conhecimentos, a comunicação com os outros e a promoção da nossa auto-imagem (Puyenbroeck \& Maes, 2005). Segundo os autores, subjacente à promoção do significado e valores pessoais está o confronto com memórias nostálgicas e conturbadas: ao enfrentar as suas memórias, avaliando-as sob uma perspectiva alternativa, o idoso resolve eventuais conflitos intrapsíquicos localizados no passado.

A reminiscência, enquanto processo normativo de evocação de acontecimentos autobiográficos inerente ao ser humano, tem diferentes objectivos. Webster $(1997,1999)$ defende serem sete as funções da reminiscência e especificamente, (i) redução de aborrecimento, (ii) preparação para a morte, (iii) resolução de problemas, (iv) conversação, (v) manutenção da intimidade, (vi) revivalismo amargurado e, por último, (vii) ensinar - informar. $\mathrm{O}$ autor considera assim que estas são as funções primordiais da reminiscência, relacionadas com parâmetros desenvolvimentais, de personalidade, e até mesmo, de género (Webster, 2001).

Puyenbroeck e Maes (2005) postulam uma tipologia da reminiscência bastante interessante, com seis variantes com finalidades diferentes, especificamente a narrativa tem como propósito contar a história de vida, a integrativa é uma metodologia de auto-avaliação, para integração das experiências passadas, a instrumental despoleta a evocação da resolução bem-sucedida de problemas, a transmissiva persegue o objectivo de passar aos outros crenças e valores significativos, a defensiva idealiza o passado, e a obsessiva descreve o estado de incapacidade para ultrapassar determinadas experiências traumáticas. São as variantes da reminiscência defensiva e obsessiva da reminiscência que estão associadas a resultados negativos, no sentido em que condicionam a adaptação ao novo momento de vida que o idoso experiencia (Puyenbroeck \& Maes, 2005).

A evocação guiada de acontecimentos de vida decorridos no passado, enquanto estratégia terapêutica estruturada para alcançar a integridade do ego, foi sugerida inicialmente por Butler, em 1963 (apud Wang, Hsu \& Cheng, 2005). Os clientes eram encorajados a falar sobre os eventos de vida ocorridos no passado que considerassem significativos, sendo auxiliados por objectos, como fotografias, vídeos ou músicas, ou por temas específicos, como "primeiro dia de aulas" ou "nascimento dos filhos" (Wang, 2005). Segundo Butler, a avaliação retrospectiva dos acontecimentos de vida permitia realizar um balanço das experiências significativas, resolvendo potenciais conflitos (Butler, 1963).

Não sendo uma estratégia exclusiva da intervenção com idosos, a eficácia da reminiscência é potenciada junto desta faixa etária, uma vez que consiste na análise do percurso de vida, realizando um balanço de vida e, eventualmente, resolvendo conflitos localizados no passado. A introdução de representações metafóricas das histórias de vida, como poemas ou desenhos, permite introduzir um elemento simbólico e aumentar o significado que tem para o sujeito (Bohlmeijer, Valenkamp, Westerhof, Smit \& Cuijpers, 2005; Haight et al., 2003).

A reminiscência, utilizada como técnica de evocação guiada, pode perseguir diferentes objectivos, consoante as características da população a que se destina. Numa perspectiva de intervenção primária, a TR pode ser utilizada para fomentar a adaptação a transições de vida, promover a autoestima e a auto-percepção de saúde, aumentar o bemestar e a satisfação de vida (Arkoff, Meredith \& Dubanoski, 2004; Cappeliez, O'Rourke \& Chaudhury, 2005; Jonsdottir et al., 2001; Wang et al., 2005; Hanaoka \& Okamura, 2004), fomentar os sentimentos de mestria, amplificar a flexibilidade no funcionamento quotidiano e prevenir o surgimento de sintomatologia depressiva (Stinson \& Kirk, 2006; Lin, Dal \& Hwang, 2003; Jones \& Beck-Little, 2002; Bohlmeijer et al., 2005; Watt, \& Cappeliez, 2000). Em quadros de funcionamento patológico, a TR pode ser utilizada para estimular o funcionamento cognitivo de idosos com demência, diminuir a incidência de sintomatologia pós-traumática, atenuar o isolamento social, auxiliar o processo de luto e diminuir a sintomatologia depressiva (Bohlmeijer et al., 2005; Jones \& Beck-Little, 2002; Serrano, Latorre, Gatz \& Montanes, 2005; Wang et al., 2005; Bohlmeijer, Smit, \& Cuijpers, 2003; Puyenbroeck \& Maes, 2005; Maercker, 2002; Lin et al., 2003). Adicionalmente, Haight et al. (2003) postulam que o desenvolvimento de um processo de reminiscência entre a díade cuidador-cuidado permite diminuir a percepção de sobrecarga do cuidador, em parte porque providencia um espaço para a interacção e partilha de experiências de vida, de que o cuidador não tinha conhecimento (Scott \& Clare, 2003). 
Vários estudos foram feitos com o intuito de avaliar a eficácia da reminiscência, tanto na óptica das revisões de literatura e meta-análises dos estudos existentes, como no âmbito dos estudos empíricos. Reunimos dados relativos aos estudos sobre a reminiscência enquanto estratégia utilizada no âmbito de quadros depressivos geriátricos, realizados nos últimos dez anos, salientando a investigação feita na área, relativamente a meta-análises e revisões de literatura (Tabela 1) e estudos empíricos (Tabela 2).

\section{Tabela 1 - Estudos de meta-análise e revisão de literatura representativos realizados sobre a reminiscência}

\begin{tabular}{llll}
\hline AUTORES TIPOLOGIA & $\begin{array}{l}\text { OBJETIVOS E } \\
\text { HIPÓtESES }\end{array}$ & METODOLOGIA
\end{tabular}

Bohlmeijer, Meta-análise

Smit \& $\quad(n=20)$

Cuijpers

(2003)

Frazer, Revisão de

Christensen \& literatura Griffiths

(2005)

Jones \& Beck- Revisão de Little (2002) literatura

Lin, Dal, \& Revisão de Hwang (2003) literatura

Scogin, Welsh, Meta-análise

Hanson, $\quad(n=20)$

Stump, Coates

(2005)
Avaliar eficácia reminiscência e revisão vida na depressão geriátrica

Avaliar os estudos de intervenção na depressão geriátrica, considerando a eficácia dos resultados.

Estudar a eficácia da TR em idosas em contextos rurais

Análise de investigações com TR junto de idosos com sintomatologia depressiva em contextos rurais.

Critérios de inclusão: (i) análise bases dados de estudos com "reminiscence", (ii) amostra mais de 65 anos, e (iii) resultados detalhados.Critérios de exclusão: (i) estudos com sujeitos com patologias físicas.

Critérios de inclusão: (i) dois estudos metodológicos com um mínimo de 30 participantes; (ii) a obtenção de resultados no GE superiores ao GC ou grupos de comparação; e (iiii) a obtenção de resultados equivalentes a outro tratamento baseado em evidências.
Insuficiência de estudos empíricos significativos

Forte heterogeneidade procedimental. Ausência modelo para as intervenções e instrumentos de medição. Necessidade controle de variáveis demográficas

Quatro estudos de reminiscência são baseados em evidências ( $c f$. texto) 
Bohlmeijer, Smit e Cuijpers (2003) realizaram uma meta-análise sobre a utilização da reminiscência e da revisão de vida, que definem como sendo métodos não-estigmatizantes de detecção e tratamento da sintomatologia depressiva. Pela análise dos estudos que utilizavam a reminiscência e a revisão de vida como estratégia de intervenção com idosos deprimidos, os autores comprovam a eficácia da estratégia junto de idosos com sintomatologia depressiva, especialmente quando se tratava de idosos inseridos na comunidade. Considerando os resultados obtidos, os autores postulam que a eficácia das estratégias referidas pode ser comparada com os resultados obtidos pela terapia cognitivo-comportamental e pelas intervenções medicamentosas.

Numa revisão sistemática sobre a eficácia das diversas estratégias de intervenção na depressão geriátrica, Frazer et al. (2005) consideram que existem suportes bem fundamentados para a eficácia da reminiscência na redução da sintomatologia depressiva. Numa escala de I a V (sendo o Grau de Evidência $\mathrm{V}$ a ausência total de fundamentação científica), os autores atribuem à TR o Grau de Evidência I. Segundo a análise proposta pelos autores, a TR possui já um corpo consistente de fundamentos quanto à sua eficácia, comprovada por diversos estudos. Tendo compartimentado as intervenções possíveis na depressão geriátrica em três áreas (medicamentosa, psicológica e complementares ou alternativas), os autores atribuíram o mesmo Grau de Evidência I à terapia cognitiva-comportamental, à intervenção medicamentosa com antidepressivos e à terapia electroconvulsiva.

Numa revisão sistemática da literatura sobre a eficácia da TR na intervenção junto com pessoas idosos, Lin, Dal \& Hwang (2003) concluíram que a dispersão de procedimentos utilizados, constatados principalmente no número variável de sessões e sua duração, e a ausência de instrumentos de avaliação sensíveis aos resultados obtidos neste tipo de intervenção prejudicam a comparação dos estudos e a promoção da eficácia da estratégia. Os autores defendem também a necessidade de desenvolver estudos que controlem as variáveis demográficas, como género e idade e, adicionalmente, propõem um modelo de enquadramento teórico, que permita alguma stardadização nos estudos de intervenção na depressão geriátrica realizada através da TR, nomeadamente em termos dos instrumentos de avaliação. Este modelo é composto por cinco fases (i) listagem dos antecedentes do idoso, (ii) avaliação, (iii) estabelecimento dos objectivos terapêuticos, (iv) selecção da modalidade e estratégias específicas de reminiscência e, por último, (v) avaliação dos resultados. Os autores postulam que a adopção da homogeneização e controlo de algumas variáveis neste tipo de estudos aumentará a sua compreensão sobre o funcionamento e a utilidade da reminiscência (Lin et al., 2003).

Com o intuito de estabelecer uma listagem das terapias para a depressão geriátrica baseadas em evidências, Scogin et al. (2005) realizaram uma pesquisa de várias fontes (bases de dados on-line, pesquisa manual, contacto directo com investigadores). Após análise de 116 estudos de intervenção na depressão geriátrica, consideraram que apenas 20 respondiam aos critérios estabelecidos para terapia baseada em evidências. Destes 20 estudos citados por Scogin et al. (2005), 4 estudos indicam que a reminiscência é uma terapia baseada em evidências para a depressão geriátrica, e especificamente se referia aos estudos de Areán et al. (1993), Goldwasser, Auerbach e Harkins (1987), Serrano et al., (2004), e por último, o de Watt \& Cappeliez (2000). Os resultados obtidos indicam que a reminiscência é uma terapia para a depressão geriátrica baseada em evidências, a nível da eficácia de outras terapias, como a Terapia Comportamental, a Terapia de Resolução de Problemas e a Terapia Psicodinâmica Breve. 


\section{Tabela 2 - Estudos empíricos representativos realizados sobre a reminiscência}

\begin{tabular}{lll}
\hline AUTORES & $\begin{array}{l}\text { TIPOLOGIA } \\
\text { E AMOSTRA }\end{array}$ & OBJETIVOS E HIPÓTESES \\
\hline $\begin{array}{l}\text { Arkoff, } \\
\text { Meredith \& }\end{array}$ & $\begin{array}{l}\text { inseridas em } \\
\text { programas de } \\
\text { Dubanoski }\end{array}$ & $\begin{array}{l}\text { Avaliar a eficácia do programa } \\
\text { The Illuminated Life para } \\
\text { promoção da adaptação às } \\
\text { (2004) }\end{array}$ \\
& $\begin{array}{l}\text { transições de vida } \\
\end{array}$ &
\end{tabular}

Bohlmeijer,

Valenkamp,

Westerhof, Smit

$\&$ Cuijpers

(2005)

$\begin{array}{ll}\text { Cappeliez, } & 420 \text { idosos a } \\ \text { O’Rourke \& } & \text { viver na } \\ \text { Chaudhury } & \text { comunidade } \\ (2005) & (\mathrm{x}=61 \text { anos })\end{array}$

Chao, Jin, Clark, Chu, Liu, Wu, \& Huang (2006)

24 idosos a viver em instituições $(\mathrm{x}=80$ anos $)$
Avaliar a eficácia do programa Searching for meaning in life, na diminuição da depressão, aumento de mestria e significado de vida

Analisar a relação entre (i)
funções da reminiscência, (ii)
traços de personalidade e (iii)
satisfação de vida e saúde mental

Avaliar o impacto da TR em grupo na DG, satisfação de vida e auto-estima de idosos institucionalizados

\author{
1. Pré-teste: Avaliação do bem- \\ estar psicológico. 2. Intervenção: \\ 14 sessões em grupo, 120 \\ minutos, temáticas \\ significativas. 3. Pós-teste
}

1. Pré-teste: Avaliação da depressão, mestria e significado de vida. 2 . Intervenção: 12 sessões em grupo, 150 minutos, temáticas predefinidas. 3. Pós-teste

1. Avaliação da personalidade, funções da reminiscência, satisfação de vida e saúde mental. 2. Análise dos resultados

$\begin{array}{lll}\text { Jones } & 30 \text { idosas a } & \text { Comparar a eficácia da TR } \\ \text { (2003) } & \text { viver em } & \begin{array}{l}\text { convencional com uma versão } \\ \text { instituições, } \\ \text { desenvolvida para técnicos de } \\ \text { enfermagem (NIC) }\end{array}\end{array}$

Serrano,

LaTorre,

Gatz \&

Montañes

(2004)

Stinson \&

Kirk (2006)
43 idosos com Avaliar a eficácia da revisão de sintomas depressivos e ausência de demência, a receber apoio domiciliário vida na diminuição da sintomatologia depressiva em idosos
24 idosos
(72-96 anos)

\section{Pré-teste: Avaliação da} depressão, satisfação de vida e auto-estima. 2. Intervenção: 9 sessões em grupo, com temáticas predefinidas. 3. Pós-teste

1. Pré-teste: Avaliação de demência e estado humor 2. Intervenção: 6 sessões, 45 minutos(GE: TR NIC; GC: TR convencional). 3. Pós-teste

1. Pré-teste: Avaliação da depressão, satisfação de vida, desesperança e memória autobiográfica. 2. Intervenção: 4 sessões individuais, através da revisão de vida. 3. Pós-teste

1. Pré-teste: Avaliação do estado de ânimo e autotranscendência. 2. Intervenção: 12 sessões grupo (GE: TR; GC: actividade). 3. Pós-teste

\section{CONCLUSÕES}

Resultados estatisticamente significativos em todas as escalas (auto-aceitação, relacionamentos positivos, propósitos de vida, autonomia, mestria ambiental, crescimento pessoal)

Resultados estatisticamente significativos na escala de depressão e de mestria Idosos com sintomatologia mais severa apresentam maiores benefícios com a intervenção

Relação estatisticamente significativa entre as funções da reminiscência e a saúde mental e o distress nas pessoas idosas. Reminiscência eficaz como meio de preparação para a morte

Resultados estatisticamente significativos na auto-estima. Resultados satisfatórios mas não significativos sob ponto de vista estatístico na depressão e satisfação de vida. Intervenção útil para aumentar interacção entre idosos

GE demonstrou resultados estatisticamente significativos na redução da sintomatologia depressiva. GC demonstrou resultados inferiores ao GE

GE evocava maior número de memórias específicas, apresentando níveis mais elevados de satisfação de vida e diminuição da sintomatologia depressiva e impotência

GE demonstrou alterações superiores ao GC, embora sem significância estatística

Depressão e autotranscendência estão inversamente correlacionadas 


$\begin{array}{lll}\text { Wang } & 48 \text { idosos } & \text { Estudar os efeitos da } \\ \text { (2005) } & \text { institucionalizados } & \text { reminiscência nos estados de } \\ & \text { a longo prazo, } & \text { humor de idosos } \\ & \text { sintomatologia } & \text { institucionalizados a longo } \\ & \text { depressiva sem } & \text { prazo } \\ \text { défices cognitivos } & \end{array}$

$\begin{array}{lll}\text { Wang } & 48 \text { idosos a } & \text { Comparar a eficácia da TR } \\ \text { (2004) } & \text { viver na } & \text { junto de idosos } \\ & \text { comunidade e } & \text { institucionalizados e a residir } \\ & \text { em instituições } & \text { na comunidade }\end{array}$

Wang, Hsu \& Cheng (2005)

\begin{abstract}
Westerhof, Bohlmeijer \& Valenkamp (2004)
\end{abstract}

$\begin{array}{ll}94 \text { idosos com } & \text { Analisar efeitos da } \\ \text { sintomas } & \text { reminiscência em quatro } \\ \text { depressivos } & \text { indicadores de saúde mental }\end{array}$

1. Pré-teste: Avaliação do estado de ânimo subjectivo e índices de observação dos investigadores. 2. Intervenção: 16 sessões individuais, 30-45 minutos, tema livre. 3. Pós-teste

\section{Pré-teste: Avaliação da} depressão, emoções aparentes e percepção de saúde2. Intervenção: 16 sessões individuais, 30-120 minutos, tema livre. 3. Pós-teste

\section{Pré-teste: Avaliação da} depressão, emoções aparentes e percepção de saúde. 2. Intervenção: 16 sessões individuais, 30-120 minutos, tema livre. 3. Pós-teste

\section{Pré-teste: Avaliação da depressão e significado pessoal. 2. Intervenção: 12 sessões em grupo; 150 minutos. 3. Pós-teste}

\author{
Resultados estatisticamente \\ significativos na diminuição da \\ sintomatologia depressiva \\ (percepção subjectiva) e \\ melhoria do estado de humor \\ (observação externa de técnicos) \\ Grupo institucionalizado \\ obteve mais resultados \\ significativos \\ Resultados significativos na \\ alteração do estado de humor \\ Grupo comunitário não \\ apresentou diferenças \\ significativas no pré e pós-teste
}

Resultados estatisticamente significativos na diminuição dos sentimentos percebidos de depressão, aumentando a autoconfiança e os sentimentos de adequação

Resultados estatisticamente significativos na escala de depressão. Idosos apresentavam menos atitudes negativas em relação ao self e às interações sociais
Tal como referido anteriormente, os estudos realizados com o intuito de avaliar a eficácia da reminiscência enquanto estratégia de intervenção na sintomatologia depressiva das pessoas idosas variam quanto ao tamanho e características da amostra (idosos institucionalizados ou inseridos na comunidade), número e duração das sessões, temáticas analisadas nestas (desde momentos específicos até todo o ciclo de vida) e modalidade de intervenção (individual ou em grupo). Vamos apresentar em seguida alguns dos estudos referidos na Tabela 2, mostrando a variedade procedimental referida; circunstância desta área de investigação descrita perfeitamente por Lin et al. (2003).

Bohlmeijer et al. (2005) desenvolveram o programa de intervenção em grupo Searching for the meaning in life, com 12 sessões de 150 minutos cada, contemplando todo o ciclo de vida de forma sucessiva (infância/adolescência; idade adulta; presente e futuro). Os dados indicam que as pessoas idosas $(\mathrm{n}=79)$ apresentaram um decréscimo significativo nos níveis da sintomatologia depressiva avaliada, e ganhos no significado de vida e sentimento de mestria, sendo os melhores resultados obtidos pelas pessoas idosas que apresentavam maior gravidade de sintomas. Contudo, os resultados finais ficaram aquém da população não-clínica. Os autores postulam que a inclusão nas sessões de tempo para avaliação e debate das memórias surgidas permitiria melhorar os resultados obtidos, pois tornaria explícita a integração das memórias passadas no momento de vida presente do idoso, o que não foi possível no formato actual de intervenção (Bohlmeijer et al., 2005).

Cappeliez et al. (2005) também defendem a eficácia da TR, postulando que o processo e as suas diferentes formas estão relacionados com a saúde mental das pessoas idosas, ao promover o bem-estar e a satisfação de vida. Contudo, este trabalho é dos raros estudos que alertam para os efeitos negativos da TR, que podem surgir consoante os conteúdos que o sujeito elicita. Assim, segundo a proposta dos autores, apresentada anteriormente, a função de continuidade que está associada à reminiscência pode traduzir-se em reminiscência integrativa (revisão de vida, balanceando aspectos negativos e positivos) ou reminiscência obsessiva 
(ruminação constante sobre eventos negativos) e de reminiscência de evitamento (fuga das recordações passadas). Nesse sentido os autores concluem, através de um estudo com 420 idosos, que a reminiscência é eficaz enquanto estratégia de preparação para a morte, ao permitir a revisão de vida, a compreensão das experiências vividas e a integração dos seus significados (Cappeliez et al., 2005).

Jones (2003) realizou um estudo com mulheres idosas institucionalizadas por um período superior a 3 meses. $\mathrm{O}$ autor pretendia comparar a eficácia terapêutica entre a terapia da reminiscência convencional com a terapia da reminiscência desenvolvida por profissionais de enfermagem, designada como Nursing Interventions Classification (NIC - Código 4860). A característica mais saliente da NIC consiste na estruturação prévia da intervenção, recorrendo a acontecimentos sociais (e.g., lançamento de uma nave espacial) e pessoais (e.g., experiências de trabalho), numa sequência cronológica. A amostra, constituída por 30 mulheres com mais de 65 anos, foi submetida a 6 sessões, com aproximadamente 30 minutos cada, ao longo de 3 semanas. O Grupo de Controlo foi submetido à terapia da reminiscência convencional e o GE foi submetido à NIC. Segundo Jones (2003), a terapia de reminiscência foi administrada num formato convencional, abordando especificamente acontecimentos recentes. Os resultados indicam que o GE obteve resultados mais significativos e pelo tanto a demonstrada a superioridade da TR do Grupo de NIC.

Wang et al. (2005) desenvolveram um programa de 16 sessões, de administração individual, junto de idosos institucionalizados e inseridos na comunidade. Após recolha inicial de dados para o pré-teste, as pessoas idosas do GE $(n=46)$ foram submetidas ao programa de intervenção e as pessoas idosas do GC ( $\mathrm{n}=48$ ) não tiveram qualquer outro contacto com os investigadores. Os resultados indicam que, em comparação com o GC, as pessoas idosas do GE manifestaram menos sintomatologia depressiva, mais autoconfiança e maiores sentimentos de adequação ao contexto onde se inseriam. Com base nos resultados obtidos, os autores propõem a utilização da terapia da reminiscência para a promoção da adaptação a novos contextos de vida, pois permite às pessoas idosas a expressão de afectos e a resolução de conflitos passados.

Westerhof, Bohlmeijer e Valenkamp (2004) utilizaram também o programa Searching for the meaning in life, com o intuito de promover o significado pessoal das pessoas idosas, nas suas componentes cognitiva (interpretação dos acontecimentos, é suportada pelas crenças pessoais e busca a manutenção da consistência interna) e motivacional (objectivos de vida que impelem o sujeito). Após intervenção junto de 57 idosos, utentes de centros comunitários de saúde mental, através de 12 sessões de 150 minutos cada, concluíram que as pessoas idosas manifestavam menos atitudes negativas em relação ao self e aos outros, participando em mais reuniões de grupo. Uma vez mais, os autores constataram que as pessoas idosas que demonstraram maiores ganhos foram aqueles que tinham pontuado mais alto nas escalas de sintomatologia depressiva.

\section{Conclusões}

Após analisar o tópico de eficácia da TR enquanto estratégia de intervenção na depressão em pessoas idosas, é possível extrair-se três linhas de conclusões acerca dos mais recentes desenvolvimentos teóricos e de investigação científica nesta área, assim como identificar as áreas que necessitam de maior pesquisa e desenvolvimento. A primeira questão relevante está relacionada com o esclarecimento sobre a eficácia da TR, isto é, falta determinar quais os mecanismos de mudança subjacentes que explicam a eficácia (ou não) da TR. Neste ponto particular, é muito interessante uma linha de investigação, oriunda da psicologia cognitiva, sobre a relação existente entre memórias autobiográficas e a sintomatologia depressiva. Em segundo lugar, existe uma segunda linha de investigação cujo objectivo é avaliar como os contextos em que se realiza a TR condiciona os resultados da mesma. Neste âmbito, parecem especialmente promissores os estudos comparativos entre eficácia de TR em contexto comunitário e em contexto institucional. Em terceiro lugar, a TR pode ter efeitos não desejados e negativos para as pessoas idosas e, neste sentido, é necessário entender as variáveis associadas com estes efeitos, como as características do terapeuta ou da pessoa idosa, que levam a que TR não produza os efeitos positivos procurados.

Relativamente aos processos de mudança associados aos resultados obtidos com a TR, estes parecem localizar-se sobretudo a nível da memória autobiográfica. Esta consiste na memória para episódios específicos do passado do sujeito, contendo uma narrativa verbal, imagens relacionadas com detalhes do acontecimento e emoções (Rubin, 1996). 
Ou seja, dizem-se autobiográficas todas as memórias com significado pessoal recuperadas pelos sujeitos, com exclusão das memórias semânticas (Swales, Williams \& Wood, 2001). As funções das memórias autobiográficas podem ser agrupadas em três blocos gerais: (i) funções do self, como regulação do autoconceito e manutenção da consistência intrapessoal, (ii) funções sociais, destacando-se o estabelecimento de relações e transmissão de valores e (iii) funções directivas, através do planeamento de decisões futuras com base em experiências passadas (Webster, 2003). Considerando as funções descritas, é possível antecipar que qualquer défice na recuperação da memória autobiográfica possa ter consequências para o sujeitos e o seu funcionamento, sendo mais documentadas na literatura a dificuldade em reformular eventos e planear as decisões (Harvey, Watkins, Mansell, \& Shafran 2004), e a iniciação e manutenção da sintomatologia depressiva (Williams, \& Broadbent, 1986).

Neste sentido, diversos autores têm explorado a relação entre memória autobiográfica e depressão (Dalgleish, Spinks, Yend, \& Kuyken, 2001; Mackinger, et al. 2004). Conquanto haja diferentes variáveis em estudo, aquela que parece colher mais consenso entre investigadores é a hipótese da sobregeneralização, que defende que perante sintomatologia específica o sujeito tem maior dificuldade em recuperar memórias autobiográficas específicas, com a duração inferior a um dia (e.g., Kuyken \& Howell, 2006; Williams, 2006). Em função das alterações da capacidade de memória inerentes ao processo de envelhecimento, nomeadamente o declínio verificado ao nível do funcionamento do córtice pré-frontal, e consequente diminuição do executivo central, as pessoas idosas tornam-se um grupo de maior vulnerabilidade ao desenvolvimento de sintomatologia depressiva, uma vez que têm maior dificuldade em recuperar memórias autobiográficas específicas, em comparação com faixas etárias mais jovens (Serrano, Latorre, Gatz \& Montañes, 2004; Levine, Svoboda, Hay, Winocur \& Moscovitch, 2002; Robertson, Myerson \& Hale, 2006).

Ainda que as estratégias de recuperação da memória autobiográfica sejam habitualmente designadas como características estáveis dos sujeitos (Peeters, Wessel, Merckelbach \& Boon-Vermeeren, 2002), estudos actuais indicam que a memória autobiográfica pode ser trabalhada, no sentido de promover e facilitar o acesso às situações de conhecimento específico. Serrano et al. (2004) realizaram uma investigação junto de 46 idosos a viver em comunidade com sintomatologia depressiva. Os resultados indicam que após a estimulação da memória autobiográfica, através de técnicas de reminiscência, nomeadamente a revisão de vida estruturada, as pessoas idosas do Grupo Experimental (GE) foram capazes de gerar mais memórias específicas, em comparação com os resultados do pré-teste e com o Grupo de Controlo (GC) (Serrano et al., 2004).

Esta linha de investigação fomenta uma interessante perspectiva. $\mathrm{O}$ facto de intervir com um protocolo muito específico em termos de objectivos terapêuticos (prática em memoria autobiográfica sobre lembranças específicas, exponencialmente positivas, a partir da revisão de vida), e breve (4 semanas), e mostrar efeitos tão eficazes pode ser uma forma de demonstrar que a TR funciona principalmente porque permite a diminuição do processo da sobregeneralização.

Em segundo lugar, parece importante o contexto em que se realiza a terapia da reminiscência, particularmente porque o formato que esta pode assumir pode ser bastante diversificado; este é um ponto importante, com implicações na gestão de serviços de saúde mental e sociais. A reminiscência pode ser realizada em contextos institucionais ou comunitários; de forma individual ou grupal, podendo ainda variar o número de elementos; com temas previamente definidos ou de modo espontâneo; analisando o percurso de vida de forma sucessiva ou explorando apenas episódios significativos isolados (Arkoff et al., 2004; Head, Portnoy \& Woods 1990; Bohlmeijer et al., 2003; Wang, 2004; Serrano et al., 2004; Chao et al., 2006; Burnside, 1995; Haight et al., 2003 ). O formato da terapia pode ainda variar quanto ao número de sessões e à duração delas.

Perante a diversidade de variáveis, o processo de compreensão de eficácia da intervenção torna-se muito complexo e enviesado. Contudo, alguns dados apontam para a superioridade da eficácia da terapia da reminiscência quando processada em contextos comunitários (Bohlmeijer et al., 2003). Estes resultados podem relacionar-se com o nível prévio de competências cognitivas por 
parte das pessoas idosas que ainda vivem em comunidade, competências estas que estão habitualmente mais deterioradas nas pessoas idosas que se encontram em situação de institucionalizações a longo prazo. Contudo, existem resultados contrários em alguns estudos, como no de Wang et al. (2005). Neste sentido, subjaz a necessidade de realização de mais investigação neste âmbito, nomeadamente em perceber as variáveis de contexto associadas com o sucesso terapêutico.

Por último, é necessário estar alerta para as consequências negativas que podem advir da utilização de estratégias de reminiscência. Tal como noutros sectores da intervenção psicossocial, a terapia da reminiscência é por vezes utilizada com base na premissa "mal não pode fazer", ou seja, considera-se que os resultados serão sempre benéficos para o idoso. Contudo, os efeitos adversos da reminiscência existem e podem surgir caso o técnico não estabeleça de modo explícito quais os seus objectivos e as metodologias que vai utilizar para os tentar alcançar (Haight, 1995; Merriam, 1995).

Neste âmbito, Cappeliez et al. (2005) postulam a existência de cinco tipos de reminiscência (narrativa, integrativa, obsessiva, de evitamento e de preocupação/preparação para a morte), relacionadas de modo distinto com a saúde mental e a promoção de adaptação à transição. Segundo os autores, a utilização da reminiscência para estimulação das pessoas idosas e para comunicação com entes do passado é prejudicial para as pessoas idosas. Em resumo, os efeitos negativos da reminiscência existem e são muitas vezes desvalorizados pelos técnicos e profissionais. Contudo, do mesmo modo que os resultados da reminiscência são validados por estudos empíricos, também os seus efeitos adversos devem ser tidos em conta.

Nos estudos de avaliação de eficácia terapêutica, como os que foram examinados, o progressivo controlo de variáveis nunca é suficiente, principalmente porque não é exequível e porque, e é mais importante, o que tem realmente faltado nesta área, são modelos teóricos que expliquem a grande diversidade de resultados, e que orientem investigação, sobretudo aquela que possa fazer uma ponte que o conhecimento clínico e as abordagens cognitivas, e neurocognitivas, para poder identificar os mecanismos de mudança que parecem se registrar na TR.

\section{Referências}

Aberg, A., Sidenvall, B., Hepworth, M., O'Reilly, K., \& Lithell, H. (2005). On loss of activity and independence, adaptation improves life satisfaction in old age - a qualitative study of patients' perceptions. Quality of Life Research, 14, 1111-1125.

American Psychiatric Association (2004). Guidelines for psychological practice with older adults. American Psychologist, 59(4), 236-260.

Arean, P. A., Perri, M. G., Nezu, A. M., Schein, R. L., Christopher, F., \& Joseph, T. X. (1993). Comparative effectiveness of social problemsolving therapy and reminiscence therapy as treatments for depression in older adults. Journal of Consulting and Clinical Psychology, 61(6), 1003-1010.

Arkoff, A., Meredith, G., \& Dubanoski, J. (2004). Gains in well-being achieved through retrospective-proactive life review by independent older women. Journal of Humanistic Psychology, 44(2), 204-214.

Baldwin, R., \& Wild, R. (2004). Management of depression in later life. Advances in Psychiatric Treatment, 10, 131-139.

Beaver, M. L., \& Miller, D. A. (1998). La práctica clínica del trabajo social con las personas mayores. Barcelona: Paidós.

Belsky, J. (1999). Psicología del envejecimiento. Madrid: International Thompson.

Bergdahl, E., Gustavsson, J., Kallin, K., Wagert, P., Lundman, B., Bucht, G., et al. (2005). Depression among the oldest old: The Umea 85+ study. International Psychogeriatrics. 17(4), 557-575.

Blazer, D. G. (2002a). Depression in late life. New York: Springer Publishing Company.

Blazer, D. G. (2002b). Self-efficacy and depression in late life: a primary intervention proposal. Aging and Mental Health, 6(4), 315-324.

Blazer, D. G. (2003). Depression in late life: review and commentary. Journal of Gerontology: Medical Sciences, 58(53), 249-265. 
Bohlmeijer, E., Smit, F., \& Cuijpers, P. (2003). Effects of reminiscence and life review on late-life depression: Ameta-analysis. International Journal of Geriatric Psychiatry, 18(12), 1088-1094.

Bohlmeijer, E., Valenkamp, M., Westerhof, G., Smit, F. \& Cuijpers, P. (2005). Creative reminiscence as an early intervention for depression: Results of a pilot study. Aging \& Mental Health, 9(4), 302-304.

Bowling, A., \& Farquhar, M. (1996). Outcome of anxiety and depression at two and a half years after baseline interview: associations with changes in psychiatric morbidity among three samples of elderly people living at home. International Journal of Geriatric Psychiatry, 11(2), 119-129.

Burnside, I. (1995). Themes in reminiscence groups with older women. In J. Hendricks (Org.). The meaning of reminiscence and life review (pp. 159-171). New York: Baywood Publishing Company.

Butler, R. N. (1963). Life review: An interpretation of reminiscence in aged. Psychiatry, 26(1), 65-76.

Cappeliez, P., O'Rourke, N., \& Chaudhury, H. (2005). Functions of reminiscence and mental health in later life. Aging and Mental Health, 9(4), 295-301.

Chao, S. Y., Jin, S. F., Clark, M. J., Chu, T. L., Liu, H. Y., Wu, C-Y., et al. (2006). The effects of group reminiscence therapy on depression, self esteem, and life satisfaction of elderly nursing home residents. Journal of Nursing Research, 14(1), 36-45.

Costa, A. (2005). A depressão nas pessoas idosas portugueses. In: C. Paúl \& A. Fonseca (Org.). Envelhecer em Portugal: Psicologia, saúde e prestação de cuidados, (pp. 159-176). Lisboa: Climepsi.

Dalgleish, T., Spinks, H., Yiend, J., \& Kuyken, W. (2001). Autobiographical memory style in seasonal affective disorder and its relationship to future symptom remission. Journal of Abnormal Psychology, 110(2), 335-340.

Douglas, S., James, I., \& Ballard, C. (2004). Nonpharmacological interventions in dementia. Advances in Psychiatric Treatment, 10, 171-177.
Form, A. F. (2000). Does old age reduce the risk of anxiety and depression? A review of epidemiological studies across the life span. Psychological Medicine, 30(1), 11-22.

Frazer, C., Christensen, H., \& Griffiths, K. (2005). Effectiveness of treatments for depression in older people. Medical Journal of Austrália, 182(12), 627-632.

Goldwasser, A. N., Auerbach, S. M., \& Harkins, S. W. (1987). Cognitive, affective, and behavioral effects of reminiscence group therapy on demented elderly. International Journal of Aging \& Human Development, 25(3), 209-222.

Grasel, E., Wilftang, J., \& Kornhuber, J. (2003). Non-drug therapies for dementia: An overview of the current situation with regard to proof of effectiveness. Dementia Geriatric Cognitive Disorders, 15(3), 115-125.

Haight, B. K. (1995). Reminiscing: The state of the art as a basis for practice. In: J. Hendricks (Org.). The meaning of reminiscence and life review (pp. 21-52). New York: Baywood Publishing Company.

Haight, B., Bachman, D., Hendrix, S., Wagner, M., Meeks, A., \& Johnson, J. (2003). Life review: Treating the dyadic family unit with dementia. Clinical Psychology and Psychotherapy, 10(3), 165-174.

Hanaoka, H., \& Okamura, H. (2004). Study on effects of Life Review activities on the quality of life of the elderly: A randomized controlled trial. Psychotherapy and Psychosomatics, 73(5), 302-311.

Harvey, A., Watkins, E., Mansell, W., \& Shafran, R. (2004). Cognitive behavioural processes across psychological disorders: Atransdiagnostic approach to research and treatment. London: Oxford Press.

Head, D. M., Portnoy, S., \& Woods, R. (1990). The impact of reminiscence groups in two different settings. International Journal of Geriatric Psychiatry, 5(5), 295-302.

Hepple, J. (2004). Psychotherapies with older people: An overview. Advances in Psychiatric Treatment, 10(1), 371-377. 
INE (2002). O envelhecimento em Portugal. Lisboa: Serviço de Estudos para a População do Departamento de Estatísticas Censitárias e de População.

Jones, E. D. (2003). Examination of reminiscence therapy for depressed older women residing in a long-term care facility using The Nursing Intervention Classification. Journal of Gerontological Nursing, 29(7), 27-33.

Jones, E. D., \& Beck-Little, R. (2002). The use of reminiscence therapy for the treatment of depression in rural-dwelling older adults. Issues in Mental Health Nursing, 23(3), 279-290.

Jonsdottir, H., Steingrimsdottir, E., \& Tryggvadottir, B. (2001). Group reminiscence among people with end-stage chronic lung diseases. Issues and Innovations in Nursing Practice, 35(1), 79-87.

Katona, C. \& Shankar, K. (1999). Depression in old age. Reviews in Clinical Gerontology, 9(4), 343-361.

Katona, C., \& Shankar, K. (2004). Depression in old age. Reviews in Clinical Gerontology, 14(4), 283-306.

Kuyken, W. \& Howell, R. (2006). Facets of autobiographical memory in adolescents with major depressive disorder and never-depressed controls. Cognition and Emotion. 20(3/4), 466-487.

Laidlaw, K. (2001). An empirical review of cognitive therapy for late life depression: Does research evidence suggest adaptations are necessary for cognitive therapy with older adults. Clinical Psychology and Psychotherapy, 8(1), 1-14.

Levine, B., Svoboda, E., Hay, J.F., Winocur, G., \& Moscovitch, M. (2002). Aging and autobiographical memory: dissociating episodic from semantic retrieval. Psychology of Aging, 17(4):677-89

Lin, Y., Dal, Y., \& Hwang, S. L. (2003). The effect of reminiscence on the elderly population: A systematic review. Public Health Nursing, 20(4), 297-306.

Mackinger, H.F., Leibetseder, M.F., Kunz-Dorfer, A., Fartacek, R., Whitworth, A.B., \& Feldinger, F.F. (2004). Autobiographical memory predicts the course of depression during detoxification therapy in alcohol dependent men. Journal of Affective Disorders. 78, 61-65.
Maercker, A. (2002). Life review technique in the treatment of PTSD in elderly patients: Rational and three single case studies. Journal of Clinical Geropsychology, 8(3), 239-249.

Merriam, S. B. (1995). Butler's life review: How universal is it? In: J. Hendricks (Org.), The meaning of reminiscence and life review, (pp. 7-20). New York: Baywood Publishing Company.

Mulsant, B., Houck, P., Gildengers, A., Andreescu, C., Dew, M., Pollock, B., et al. (2006). What is the optimal duration of a short-term antidepressant trial when treating geriatric depression? Journal of Clinical Psychopharmacology, 26(2), 113-121.

Murphy, S. (2000). Provision of psychotherapy services for older people. Psychiatric Bulletin, 24(1), 181-184.

Peeters, F., Wessel, I., Merckelbach H., \& BoonVermeeren, M. (2002). Autobiographical memory specificity and the course of major depressive disorder. Comprehensive Psychiatry, 43(5), 344-350.

Puyenbroeck, J., \& Maes, B. (2005). Reminiscence in ageing people with intellectual disabilities: An exploratory study. British Journal of Developmental Disabilities, 51(1) 3-16.

Robertson, S., Myerson, J., \& Hale, S. (2006). Are there age differences in intraindividual variability in working memory performance? Journal of Gerontology Psychological Sciences. 61B(1), 18-24.

Rubin, D.C. (1996). Introduction. In D.C. Rubin (Ed.). Remembering our past: Studies in autobiographical memory. (pp. 1-15). New York: Cambridge University Press.

Scogin, F., Welsh, D., Hanson, A., Stump, J., \& Coates, A. (2005). Evidence-Based Psychoterapies for Depression in Older Adults. Clinical Psychology - Science and Practice, 12(3), 222-237.

Scott, J. \& Clare, L. (2003). Do people with dementia benefit from interventions offered on a group basis? Clinical Psychology and Psychotherapy, 10(3), 186-196. 
Serrano, J. P.; LaTorre, J. M.; Gatz, M., \& Montanes, J. (2004). Life review using autobiographical retrieval practice for older adults with depressive symptomatology. Psychology and Aging, 19(2), 272-277.

Serrano, J. P.; Latorre, J. M., \& Montañes, J. (2005). Terapia sobre revisión de vida basada en la recuperación de recuerdos autobiográficos específicos en ancianos que presentan síntomas depresivos. Revista Española de Geriatría y Gerontologia, 40(4), 228-235.

Shah, A., \& Hoxey, K. (2001). Depression in acutely medically ill elderly inpatients: Prevalence, correlates and longitudinal stability. International Journal of Methods in Psychiatric Research, 10(3), 147-156.

Stinson, C. K., \& Kirk, E. (2006). Structured reminiscence: an intervention to decrease depression and increase self-transcendence in older women. Journal of Clinical Nursing, 15(2), 208-218.

Swales, M. A.; Williams, J. M., \& Wood, P. (2001). Specificity of autobiographical memory and mood disturbance in adolescents. Cognition and Emotion, 15(3), 321-331.

Taylor, W. D., \& Doraiswamy, P. M. (2004). A systematic review of antidepressant placebocontrolled trials for geriatric depression: Limitations of current data and directions for the future. Neuropsychopharmacology, 29(12), 2285-2299.

Van-Vreeswijk, M., \& De-Wilde, E. (2004). Autobiographical memory specificity, psychopathology, depressed mood and the use of Autobiographical Memory Test: A metaanalysis. Behaviour Research and Therapy, 42(6), 731-743.

Voyer, P., \& Martin, L. S. (2003). Improving geriatric mental health nursing care: Making a case for going beyond psychotropic medications. International Journal of Mental Health Nursing, 12(1), 11-21.

Wang, J. (2004). The comparative effectiveness among institutionalized and noninstitutionalized elderly people in Taiwan of reminiscence therapy as a psychological measure. Journal of Nursing Research, 12(3), 237-245.

Wang, J. (2005). The effects of reminiscence on depressive symptoms and mood status of older institutionalized adults in Taiwan. International Journal of Geriatric Psychiatry, 20(1), 57-62.
Wang, J., Hsu, Y., \& Cheng, S. (2005). The effects of reminiscence in promoting mental health of Taiwanese elderly. International Journal of Nursing Studies, 42(1), 31-36.

Watt, L.M. \& Cappeliez, P. (2000). Integrative and instrumental reminiscence therapies for depression in older adults: Intervention strategies and treatment eefectiveness. Aging \& Mental Health, 4(2), 166-177.

Webster, J. D. (1997). The Reminiscence Functions Scale: Areplication. International Journal of Aging and Human Development, 44(2), 136-148.

Webster, J. D. (1999). World views and narrative gerontology: Situating reminiscence behaviour within a lifespan perspective. Journal of Aging Studies, 13(1), 29-42.

Webster, J. D. (2001). The future of the past: Continuing challenges for reminiscence research. In: G. Kenyon, P. Clark, \& B. Vries (Org.), Narrative Gerontology: Theory, Research and Practice. New York: Springer Publishing Company.

Webster, J. D. (2003). The reminiscence circumplex and autobiographical memory functions. Memory, 11(2), 203-215.

Westerhof, G., Bohlmeijer, E., \& Valemkamp, M. (2004). In search of meaning: A reminiscence program for older persons. Educational Gerontology, 30(9), 751-766.

Williams, J.M.G. \& Broadbent, K. (1986). Autobiographical memory in suicide attempters. Journal of Abnormal Psychology, 95(2), 144-149.

Williams, J. M. (2006). Capture and rumination, functional avoidance, and executive control (CaRFAX): Three processes that underlie overgeneral memory. Cognition and Emotion. 20(3/4), 548-568.

Woods, R. T. (1999). Mental health problems in later life. In: R. T. Woods (Org.). Psychological problems of ageing: Assessment, treatment and care, (pp. 73-110). Chichester: John Wiley \& Sons.

\footnotetext{
Recebido em: 14/03/2007 Received in: 03/14/2007

Aprovado em: 06/04/2007 Approved in: 04/03/2007
} 